\title{
Aggregation of the nearest consistency matrices with the acceptable consensus in AHP-GDM
}

\author{
Changsheng $\mathrm{Lin}^{1} \cdot$ Gang $\mathrm{Kou}^{2} \cdot \mathrm{Yi} \mathrm{Peng}^{3} \cdot$ Fawaz E. Alsaadi ${ }^{4}$ \\ Published online: 13 March 2020 \\ (c) The Author(s) 2020
}

\begin{abstract}
Analytic hierarchy process (AHP) is widely used in group decision making (GDM). There are two traditional aggregation methods for the collective preference in AHP-GDM: aggregation of the individual judgments (AIJ) and aggregation of the individual priorities (AIP). However, AHP-GDM is sometimes less reliable only under the condition of AIJ and AIP because of the consensus and consistency of the individual pair-wise comparison matrices (PCMs) and prioritization methods. In this paper, we propose aggregation of the nearest consistent matrices (ANCM) with the acceptable consensus in AHP-GDM, simultaneously considering the consensus and consistency of the individual PCMs. ANCM is independent of prioritization methods while complying with the Pareto principal of social choice theory. Moreover, ANCM is easy to program and implement in resolving highly complex group decision making problems. Finally, two numerical examples illustrate the applications and advantages of the proposed ANCM.
\end{abstract}

Keywords Group decision making (GDM) · Pair-wise comparison matrix (PCM) · Consistency $\cdot$ Consensus $\cdot$ Nearest consistent matrix

\section{Introduction}

Group decision making (GDM) arises from the real-world situations in many fields (Li et al. 2018; Zhang et al. 2019) such as society, economy, management, etc. GDM mainly aims to

Gang Kou

kougang@swufe.edu.cn

1 School of Mathematics and Statistics, Yangtze Normal University, Chongqing 408100, China

2 School of Business Administration, Southwestern University of Finance and Economics, Chengdu 611130, China

3 School of Management and Economics, University of Electronic Science and Technology of China, Chengdu 611731, China

4 Department of Information Technology, Faculty of Computing and IT, King Abdulaziz University, Jeddah, Saudi Arabia 
obtain a collective preference by aggregating the individual preferences, and then select the available alternative among alternatives. The standard AHP-individual application has been extended to aggregation, consensus procedure, etc. (Srdjevic and Srdjevic 2013). Most of GDM problems have been solved by analytic hierarchy process (AHP) (Saaty 1977, 1980) as multiple criteria decision making (MCDM) method (Kou et al. 2012, 2014, 2016) due to its simplicity, powerfulness, and minimal data dependence.

For a common hierarchy with multiple actors in AHP-GDM, there are two traditional aggregation methods for the collective preference: aggregation of the individual judgments (AIJ) and aggregation of the individual priorities (AIP) (Aczel and Saaty 1983; Saaty 1989; Barzilai and Golany 1994; Ramanathan and Ganesh 1994; Forman and Peniwati 1998; Zahir 1999). Recently, AIJ and AIP have been widely accepted and used when the opinions of decision makers are not strongly divergent. However, AHP-GDM is sometimes less reliable only under the condition of AIJ and AIP because of the consensus and consistency of the individual pair-wise comparison matrix (PCMs) and prioritization methods. Moreover, there still are some disputes on the use of AIJ and AIP in the aggregation of the individual preferences. For example, Ramanathan and Ganesh (1994) suggested using AIP because AIJ violates the Pareto principle of social choice theory (Arrow 1963) when selecting eigenvector method (EM) as prioritization method; Forman and Peniwati (1998) argued that whether AIJ or AIP is used depends on whether the group intends to behave as a synergistic unit or as a group of individuals, respectively; Barzilai and Golany (1994) observed that there is an equivalence between AIJ and AIP when selecting logarithmic least squares method (LLSM) as prioritization method.

However, there are always some limitations and disputes on AIJ and AIP in the existing literature. To be more specific, AIJ and AIP are complemented under the assumption that the consensus exists among decision makers (Altuzarra et al. 2007; Lin and Kou 2015); The results of AIJ and AIP mainly depend on the consensus and consistency of the individual PCMs and prioritization methods. In order to overcome some limitations and disputes on AIJ and AIP, several other aggregation methods have been proposed to obtain the reliable collective preference in AHP-GDM. For example, Altuzarra et al. (2007) proposed Bayesian prioritization procedure (BPP) that provides more efficient estimates than AIJ and AIP; Escobar and Moreno-Jimenez (2007) proposed aggregation of the individual preference structures (AIPS) that provides the holistic importance of each alternative and ranking; Gargallo et al. (2007) proposed a Bayesian estimation procedure to determine the priorities of AHP in GDM; Srdjevic and Srdjevic (2013) proposed to perform an AHP synthesis of the best local priority vectors taken from the most consistent decision makers.

Moreover, lots of literature focuses on either the consistency or the consensus for the individual PCMs in AHP-GDM. For example, Lin and Kou (2015) improved the consistency levels of the individual PCMs by Bayesian approach; Altuzarra et al. (2010) proposed a Bayesian approach for building the consensus of the individual PCMs; Dong et al. (2010) proposed two consensus models to improve the consensus indices of the individual PCMs. Dong and Saaty (2014) investigated the consensus measure and consensus reaching process. Nevertheless, little literature simultaneously considers the consensus and consistency of the individual PCMs in AHP-GDM although some achievements regarding them have been made (Altuzarra et al. 2010; Dong et al. 2010; Wu and Xu 2012; Li et al. 2017). Therefore, it is preferable to take both consistency and consensus into account at the same time for the individual PCMs in AHP-GDM.

It is necessary to further investigate revising the individual PCMs for the acceptable consistency and consensus before using AIJ and AIP. For the consistency of the individual PCMs, several approaches for obtaining the nearest consistency matrix have been proposed and used 
in the actual decision making (Koczkodaj and Orlowski 1997; Benítez et al. 2011; Lin et al. 2013; Benítez et al. 2014) since the nearest consistency matrix is not concerned with prioritization method. For the consensus of the individual PCMs, Altuzarra, et al. (2007) proposed Opening Coefficient (OC) to find the judgments breaking consensus in the individual PCMs, and then omit the judgments disagreeing most with the collective preference.

In this paper, we propose aggregation of the nearest consistent matrices (ANCM) with the acceptable consensus in AHP-GDM, simultaneously considering consensus and consistency of the individual PCMs. First, we obtain the nearest consistent matrices for the inconsistent PCMs. Next, we find the judgments breaking consensus in the individual PCMs by OC, and replace them by the corresponding geometric means of the other individual judgments. Finally, we aggregate the nearest consistent matrices (ANCM) with the acceptable consensus and then obtain the group priority vector by any prioritization method. ANCM provides a flexible framework for the group priority vector while complying with the Pareto principal of social choice theory. Moreover, we describe the involved steps for obtaining the group priority vector by ANCM in AHP-GDM and assess the performance of ANCM by geometric compatibility index (GCI) and priority violation number (PVN). In the actual decision situation, ANCM is easy to program and implement, which is more practical for highly complex group decision making problems.

The rest of this paper is structured as follows. Section 2 briefly reviews the related researches such as AHP-GDM, prioritization methods, aggregation methods (AIJ and AIP), consensus, consistency and compatibility. Section 3 proposes ANCM to obtain the group priority vector. Section 4 illustrates the applications and advantages of the proposed ANCM using two numerical examples. Section 5 concludes the main results obtained from this study.

\section{Related researches}

\subsection{AHP-GDM}

AHP-GDM mainly aims to obtain the group priority vector based on the individual PCMs as it leads to a cardinal ranking of actions, objectives, attributes and criteria relevant to the actual decision situation.

AHP is one of the most popular decision support tools in GDM involving multiple actors, scenarios and criteria. First, two definitions related to the PCM in AHP are introduced as follows:

Definition 1 Matrix $A=\left(a_{i j}\right)_{n \times n}$ is said to be positive reciprocal matrix if $a_{i j}>0, a_{i i}=1$ and $a_{i j}=1 / a_{j i}$ for all $i, j \in\{1,2, \ldots, n\}$.

Definition 2 A positive reciprocal matrix $A=\left(a_{i j}\right)_{n \times n}$ is said to be consistent if $a_{i j}=a_{i l} a_{l j}$ for all $i, j, l \in\{1,2, \ldots, n\}$.

GDM is widely advocated to pool the opinions of decision makers in the actual decision situation because of its flexibility and adaptability. In this paper, we only consider a local context (a unique criterion) in GDM with a group of $r(r \geq 2)$ decision makers getting together to independently make decisions.

In AHP-GDM, according to the individual judgments for $n$ alternatives with regard to the considered criterion, each decision maker produces an individual PCM, we write, $A^{(k)}=$ $\left(a_{i j}^{(k)}\right)_{n \times n}(k=1,2, \ldots, r)$, where, $a_{i j}^{(k)}=1(i=j)$, and $a_{i j}^{(k)}(i \neq j)$ are the individual judgments provided by the $k t h$ decision maker under the condition of one to nine scale. 
Note that the individual PCMs are positive reciprocal matrices. According to Definition 1, it follows that $a_{i j}^{(k)}>0, a_{i i}^{(k)}=1$ and $a_{i j}^{(k)}=1 / a_{j i}^{(k)}$ for all $i, j \in\{1,2, \ldots, n\}(k=$ $1,2, \ldots, r)$. We further assume that $V^{(k)}=\left(v_{1}^{(k)}, v_{2}^{(k)}, \ldots, v_{n}^{(k)}\right)^{T}$ denotes the individual priority vector derived from the $k t h$ individual PCM $A^{(k)}=\left(a_{i j}^{(k)}\right)_{n \times n}(k=1,2, \ldots, r)$ by certain prioritization method, and that $V^{G}=\left(v_{1}^{G}, v_{2}^{G}, \ldots, v_{n}^{G}\right)^{T}$ denotes the group priority vector obtained by certain aggregation method.

\subsection{Prioritization methods}

Recently, some prioritization methods have been proposed and widely used in AHP, including additive normalization method (ANM) (Saaty 1977), eigenvector method (EM) (Saaty 1980), logarithmic least squares method (LLSM) (Crawford and Williams 1985), cosine maximization method (CMM) (Kou and Lin 2014), etc. Several popular prioritization methods (EM, ANM and LLSM) are briefly listed below. Assume that $A=\left(a_{i j}\right)_{n \times n}$ denotes a PCM, and that $V=\left(v_{1}, v_{2}, \ldots, v_{n}\right)^{T}$ denotes the desired priority vector derived from $A$ by certain prioritization method.

\subsubsection{Eigenvector method (EM)}

The desired priority vector $V=\left(v_{1}, v_{2}, \ldots, v_{n}\right)^{T}$ is obtained by solving the following linear system: $A V=\lambda V$ subject to $\sum_{i=1}^{n} v_{i}=1, v_{i} \geq 0(i=1,2, \ldots, n)$, where $\lambda$ is the principal eigenvalue of $A=\left(a_{i j}\right)_{n \times n}$.

\subsubsection{Additive normalization method (ANM)}

The desired priority vector $V=\left(v_{1}, v_{2}, \ldots, v_{n}\right)^{T}$ is obtained by the following procedures:

First $a_{i j}^{\prime}=a_{i j} / \sum_{i=1}^{n} a_{i j}(i, j=1,2, \ldots, n)$, and then $v_{i}=\sum_{j=1}^{n} a_{i j}^{\prime} / n(i=$ $1,2, \ldots, n)$.

\subsubsection{Logarithmic least squares method (LLSM)}

The desired priority vector $V=\left(v_{1}, v_{2}, \ldots, v_{n}\right)^{T}$ is formulated as the multiplicative normalizing constrained optimization problem:

$$
\operatorname{Min} \sum_{i=1}^{n} \sum_{j=1}^{n}\left(\ln a_{i j}-\ln v_{i}+\ln v_{j}\right)^{2} \text { subject to } \sum_{i=1}^{n} v_{i}=1, v_{i}>0(i=1,2, \ldots, n) \text {. }
$$

It is well known that different prioritization methods produce the same result for any consistent PCM. However, different prioritization methods produce different results for an inconsistent PCM. None of prioritization methods is superior to other prioritization methods according to the global criteria such as Euclidean distance and Minimum violation (Golany and Kress 1993; Srdjevic 2005).

\subsection{AIJ and AIP}

The collective preference is usually obtained by certain aggregation method based on the individual preferences in GDM. In AHP-GDM, AIJ and AIP are two traditional aggregation methods to obtain the group priority vector. 
For AIJ, first aggregate the individual judgments using weighted geometric mean method (WGMM) to obtain a group matrix $A^{G}=\left(a_{i j}^{G}\right)_{n \times n}$, where

$$
a_{i j}^{G}=\prod_{k=1}^{r}\left(a_{i j}^{(k)}\right)^{\lambda_{k}}, \quad i, j \in\{1,2, \ldots, n\}
$$

Then derive a group priority vector $V^{G}=\left(v_{1}^{G}, v_{2}^{G}, \ldots, v_{n}^{G}\right)^{T}$ from $A^{G}$ by certain prioritization method.

For AIP, first derive the individual priority vectors $V^{(k)}=\left(v_{1}^{(k)}, v_{2}^{(k)}, \cdots, v_{n}^{(k)}\right)^{T}$ from the individual PCMs $A^{(k)}(k=1,2, \ldots, r)$ by certain prioritization method. Then derive a group priority vector $V^{G}=\left(v_{1}^{G}, v_{2}^{G}, \ldots, v_{n}^{G}\right)^{T}$ using WGMM, where

$$
v_{i}^{G}=\prod_{k=1}^{r}\left(v_{i}^{(k)}\right)^{\lambda_{k}}, \quad i=1,2, \ldots, n
$$

Note that the weight vector of $r$ decision makers is $\left(\lambda_{1}, \lambda_{2}, \ldots, \lambda_{r}\right)$ with $\sum_{k=1}^{r} \lambda_{k}=1$ and $\lambda_{k}>0(k=1,2, \ldots, r)$, and that both AIJ and AIP demand that the judgments in the individual PCMs are complete and accurate.

\subsection{Consistency and consensus}

Generally, the individual PCMs are not of the acceptable consensus and consistency in AHP-GDM because the judgments expressed by decision makers depend on the personal psychological aspects such as experience, learning, situation, etc. Thus, it is necessary to consider whether the individual PCMs are of the acceptable consensus and consistency in AHP-GDM.

In order to test whether a PCM is of the acceptable consistency in AHP, Saaty (1980) proposed consistency ratio (CR) that is defined by

$$
C R=(\lambda-n) / R I(n-1)
$$

where $\lambda$ and $n$ are the principal eigenvalue and order of the PCM, respectively, and RI is the random consistency index simulated under the condition of one to nine scale. For an inconsistent PCM, CR should be as small as possible since $C R=0$ for any consistent PCM. If $0<C R<0.10$, then the PCM is of the acceptable consistency. If $C R \geq 0.10$, then a re-examination of the PCM is recommended until $C R<0.10$.

In order to measure the consensus of an individual PCM with the collective preference, Dong et al. (2010) defined geometric cardinal consensus index (GCCI) and geometric ordinal consensus index (GOCI)), which are introduced as follows:

GCCI measures the cardinal consensus of an individual PCM, which is denoted by

$$
\operatorname{GCCI}\left(A^{(k)}\right)=\frac{2}{n(n-1)} \sum_{i<j}\left[\ln \left(a_{i j}^{(k)}\right)-\ln \left(v_{i}^{G}\right)+\ln \left(v_{j}^{G}\right)\right]^{2}(k=1,2, \ldots, r)
$$

where $A^{(k)}=\left(a_{i j}^{(k)}\right)_{n \times n}$ is the $k t h$ individual PCM, and $V^{G}=\left(v_{1}^{G}, v_{2}^{G}, \ldots, v_{n}^{G}\right)^{T}$ is the group priority vector by LLSM under AIJ.

GOCI measures the ordinal consensus of an individual PCM, which is denoted by

$$
\operatorname{GOCI}\left(A^{(k)}\right)=\frac{1}{n} \sum_{i=1}^{n}\left|v_{i}^{(k)}-v_{i}^{G}\right|(k=1,2, \ldots, r)
$$


where $A^{(k)}=\left(a_{i j}^{(k)}\right)_{n \times n}$ is the $k t h$ individual PCM, and $v_{i}^{(k)}$ and $v_{i}^{G}$ are the positions of the $i t h$ alternative in $V^{(k)}$ and $V^{G}$, respectively. Here, $V^{(k)}=\left(v_{1}^{(k)}, v_{2}^{(k)}, \ldots, v_{n}^{(k)}\right)^{T}$ are the individual priority vectors derived from $A^{(k)}(k=1,2, \ldots, r)$ by LLSM, and $V^{G}=\left(v_{1}^{G}, v_{2}^{G}, \ldots, v_{n}^{G}\right)^{T}$ is the group priority vector by LLSM under AIJ.

If $G C C I\left(A^{(k)}\right)=0\left(\operatorname{GOCI}\left(A^{(k)}\right)=0\right)$, then the kth individual PCM is of fully cardinal (ordinal) consensus. If $G C C I\left(A^{(k)}\right)\left(G O C I\left(A^{(k)}\right)\right)$ is less than the established threshold according to the actual situation, then $A^{(k)}$ reaches the acceptable cardinal (ordinal) consensus; Otherwise, we revise it until $A^{(k)}$ reaches the acceptable cardinal (ordinal) consensus. Until now, the thresholds of GCCI and GOCI are not established by the existing literature. However, the smaller the value of GCCI (GOCI) is, the better the cardinal (ordinal) consensus. Without loss of generality, suppose that the acceptable threshold of GCCI is 0.5 , and that the desired value of GOCI is zero in this paper. In the actual situation, we first check and revise the individual PCMs to make GCCIs and GOCIs of them as small as possible.

\subsection{Compatibility}

In order to assess the performance of the proposed ANCM in AHP-GDM, we introduce two compatibility indicators associated with the compatibility between the individual judgments and the group priority vector: geometric compatibility index (GCI) (Moreno-Jiménez 2011) and priority violation number (PVN) (Golany and Kress 1993).

GCI is the cardinal compatibility index between the group priority vector and individual PCMs, which is defined by

$$
G C I=\sum_{k=1}^{r} \lambda_{k}\left(\frac{2}{(n-2)(n-1)} \sum_{i<j} \ln ^{2}\left(a_{i j}^{(k)} v_{j}^{G} / v_{i}^{G}\right)\right)
$$

where $\left(\lambda_{1}, \lambda_{2}, \ldots, \lambda_{r}\right)$ with $\sum_{k=1}^{r} \lambda_{k}=1$ and $\lambda_{k}>0(k=1,2, \ldots, r)$ is the weight vector of $r$ decision makers.

PVN is the ordinal compatibility index denoting the number of violations in priorities for the individual PCMs, which is defined by

$$
P V N=\sum_{k=1}^{r} \frac{2}{(n-2)(n-1)} \sum_{i<j} I_{i j}\left(A^{(k)}\right), \quad I_{i j}\left(A^{(k)}\right)=\left\{\begin{array}{c}
1 \text { if } v_{i}^{G}<v_{j}^{G} \text { and } a_{i j}>1 \\
0.5 \text { if } v_{i}^{G}=v_{j}^{G} \text { and } a_{j i} \neq 1 \\
0.5 \text { if } v_{i}^{G} \neq v_{j}^{G} \text { and } a_{j i}=1 \\
0 \text { otherwise }
\end{array}\right.
$$

Both GCI and PVN are employed to evaluate the compatibility between the group priority vector and the initial individual judgments. The smaller the values of GCI and PVN are, the better the compatibility between the individual judgments and the group priority vector.

\section{Aggregation of the nearest consistency matrices (ANCM)}

In AHP-GDM, the individual PCMs should be of the acceptable consensus and consistency before using AIJ and AIP. The nearest consistency matrix with the acceptable consensus is the best approximation of the original individual PCM. Therefore, we replace the individual PCM by its nearest consistency matrix with the acceptable consensus in AHP-GDM. 
First, we adjust the individual PCMs without the acceptable consensus until they reach the acceptable consensus. The revised individual PCM is called individual revision matrix. Then, we derive the nearest consistency matrices of the individual revision matrices and the individual PCMs with the acceptable consensus. Finally, we aggregate the nearest consistency matrices by WGMM and then derive the group priority vector by certain prioritization method.

\subsection{Revision of the consensus of the individual PCMs}

In order to find the judgments breaking consensus in the individual PCMs in AHP-GDM, we introduce opening coefficient (OC) (Altuzarra et al. 2007) as consensus tool for the individual PCMs $A^{(k)}=\left(a_{i j}^{(k)}\right)_{n \times n}(k=1,2, \ldots, r)$, which is denoted by

$$
O C_{i j}=\operatorname{Max}\left\{a_{i j}^{(k)}\right\} / \operatorname{Min}\left\{a_{i j}^{(k)}\right\}(1 \leq i<j \leq n)
$$

In particular, OC can find the judgments disagreeing with the collective preference preferred by the other decision makers. Thus, the judgments found by OC should be dealt with until the individual PCM reaches the acceptable consensus. For example, Altuzarra et al. (2007) omit the judgments disagreeing most with the collective preference in decision analysis. In this paper, we will replace the judgments disagreeing most with the collective preference by the corresponding geometric means of the other individual judgments.

\subsection{Nearest consistent matrix}

It is well known that different prioritization methods have the same results for any consistent PCM. The results of AIJ and AIP are identical, regardless of prioritization method when the individual PCMs are placed by the corresponding nearest consistency matrices in AHPGDM. We now briefly review some notations and theorems related to the nearest consistency matrix.

The set of $n \times n$ matrices and the set of $n \times n$ matrices with positive entries are denoted by $M_{n, n}$ and $M_{n, n}^{+}$, respectively. Furthermore, two inversed mappings are defined by

$$
\begin{gathered}
L: M_{n, n}^{+} \rightarrow M_{n, n},[L(X)]_{i j}=\ln \left([X]_{i j}\right), X \in M_{n, n}^{+} \\
E: M_{n, n} \rightarrow M_{n, n}^{+},[E(X)]_{i j}=\exp \left([X]_{i j}\right), X \in M_{n, n}
\end{gathered}
$$

Frobenius norm of matrix $A=\left(a_{i j}\right)_{n \times n}$ is defined by

$$
\|A\|_{F}=\left(\sum_{i=1}^{n} \sum_{j=1}^{n} a_{i j}^{2}\right)^{1 / 2}, A \in M_{n, n}
$$

Frobenius norm is an index to measure the nearness of two matrices (Meyer 2000), thus, the distance between two matrices $A$ and $B$ can be denoted by Frobenius norm as follows:

$$
d(A, B)=\|A-B\|_{F}, A, B \in M_{n, n}
$$

Theorem 1 (Meyer 2000) Let matrix $A \in M_{n, n}^{+}$, minimizing $d\left(A, A^{*}\right)=\left\|A-A^{*}\right\|_{F}$ is equivalent to minimizing $d\left(L(A), L\left(A^{*}\right)\right)=\left\|L(A)-L\left(A^{*}\right)\right\|_{F}$ for any consistent matrix $A^{*} \in M_{n, n}^{+}$.

Theorem 2 (Meyer 2000) Let matrix $A \in M_{n, n}^{+}$, then $X$ is the nearest consistency matrix of $L(A)$ if, and only if, $E(X)$ is the nearest consistency matrix of $E(L(A))=A$. 
Theorem 3 (Benítez et al. 2011) Let matrix $A \in M_{n, n}^{+}$, then there exists a unique consistent matrix $A^{*} \in M_{n, n}^{+}$, such that $\left\|L(A)-L\left(A^{*}\right)\right\|_{F} \leq\|L(A)-L(C)\|_{F}$ for any consistent matrix $C \in M_{n, n}^{+}$.

Proof of Theorems 1, 2, 3. See the references (Meyer 2000; Benítez et al. 2011)

Base on the above notations and theorems, we have developed a two-step algorithm for deriving the nearest consistency matrix of an inconsistent PCM (Lin et al. 2013), which is briefly introduced as follows:

Step 1 Calculate the transitional matrix $X=\left(x_{i j}\right)_{n \times n}$ for a PCM $A=\left(a_{i j}\right)_{n \times n}$, where $x_{i j}=\frac{1}{n} \ln \left(\prod_{l=1}^{n} a_{i l} / a_{j l}\right)(i<j), x_{i j}=-x_{j i}(i>j), x_{i i}=0$ for all $i, j \in\{1,2, \ldots, n\}$;

Step 2 Calculate the nearest consistency matrix $A^{*}=\left(a_{i j}^{*}\right)_{n \times n}$ of $A=\left(a_{i j}\right)_{n \times n}$, where $a_{i j}^{*}=\exp \left\{x_{i j}\right\}(i<j), a_{i j}^{*}=1 / a_{j i}^{*}(i>j), a_{i i}^{*}=1$ for all $i, j \in\{1,2, \ldots, n\}$.

For simplicity and consistency, we directly adopt the above two-step algorithm to derive the nearest consistency matrices of the individual PCMs in AHP-GDM.

\subsection{Group priority vector}

In AHP-GDM, we still assume that $V^{(k)}=\left(v_{1}^{(k)}, v_{2}^{(k)}, \ldots, v_{n}^{(k)}\right)^{T}$ denote the individual priority vectors derived from $A^{(k)}=\left(a_{i j}^{(k)}\right)_{n \times n}(k=1,2, \ldots, r)$ by certain prioritization method, that $V^{G}=\left(v_{1}^{G}, v_{2}^{G}, \ldots, v_{n}^{G}\right)^{T}$ denotes the group priority vector obtained by certain aggregation method, and that $\left(\lambda_{1}, \lambda_{2}, \ldots, \lambda_{r}\right)$ with $\sum_{k=1}^{r} \lambda_{k}=1$ and $\lambda_{k}>0(k=1,2, \ldots, r)$ denotes the weight vector of $r$ decision makers. Several theorems related to AHP-GDM are introduced as follows:

Theorem 4 If a PCM A $=\left(a_{i j}\right)_{n \times n}$ is consistent in AHP, and $V=\left(v_{1}, v_{2}, \ldots, v_{n}\right)^{T}$ is the priority vector derived from $A$, then $V$ is unique with $\sum_{i=1}^{n} v_{i}=1$ and $v_{i} \geq 0(i=$ $1,2, \ldots, n)$, regardless of prioritization method.

Theorem 5 Let the individual PCMs $A^{(k)}=\left(a_{i j}^{(k)}\right)_{n \times n}(k=1,2, \ldots, r)$ be consistent in AHP-GDM, then the group matrix $A^{G}=\left(a_{i j}^{G}\right)_{n \times n}=\left(\prod_{k=1}^{r}\left(a_{i j}^{(k)}\right)^{\lambda_{k}}\right)_{n \times n}$ obtained by WGMM is consistent.

Proof of Theorems 4 and 5 is straightforward, we omit it here. From Theorem 4, the priority vector derived from the nearest consistency matrix is unique, regardless of prioritization method. Therefore, any of the known prioritization methods such as EM, ANM and LLSM, can be used to derive the unique priority vector from the nearest consistency matrix. From Theorems 4 and 5, the group matrix obtained by ANCM is consistent, thereby, the group priority vector is unique, regardless of prioritization method.

Theorem 6 Let $V^{G 1}=\left(v_{1}^{G 1}, v_{2}^{G 1}, \ldots, v_{n}^{G 1}\right)^{T}$ and $V^{G 2}=\left(v_{1}^{G 2}, v_{2}^{G 2}, \ldots, v_{n}^{G 2}\right)^{T}$ be the group priority vectors in AHP-GDM through AIJ and AIP based on the nearest consistency matrices, respectively. Then $v_{i}^{G 1}=v_{i}^{G 2}$ for all $i \in\{1,2, \ldots, n\}$ with $\sum_{i=1}^{n} v_{i}^{G k}=1$ and $v_{i}^{G k} \geq 0(i=1,2, \ldots, n), k=1,2$.

Proof Assume that $A^{(k *)}=\left(a_{i j}^{(k *)}\right)_{n \times n}(k=1,2, \ldots, r)$ are the nearest consistency matrices, and that the weight vector of $r$ decision makers is $\left(\lambda_{1}, \lambda_{2}, \ldots, \lambda_{r}\right)$ with $\sum_{k=1}^{r} \lambda_{k}=1$ and $\lambda_{k}>0(k=1,2, \ldots, r)$. 
Under AIJ, aggregating the nearest consistency matrices $A^{(k *)}=\left(a_{i j}^{(k *)}\right)_{n \times n}(k=$ $1,2, \ldots, r)$ by WGMM, a group matrix is obtained as follows: $A^{G}=\left(a_{i j}^{G}\right)_{n \times n}=$ $\left(\prod_{k=1}^{r}\left(a_{i j}^{(k *)}\right)^{\lambda_{k}}\right)_{n \times n}$.

According to Theorem 5, the group matrix $A^{G}=\left(a_{i j}^{G}\right)_{n \times n}$ is consistent. According to Theorem 4 , the obtained group priority vector from $A^{G}$ by any prioritization method (Here, use LLSM) is $V^{G 1}=\left(v_{1}^{G 1}, v_{2}^{G 1}, \ldots, v_{n}^{G 1}\right)^{T}$, where

$$
v_{i}^{G 1}=\left(\prod_{j=1}^{n} a_{i j}^{G}\right)^{1 / n}=\left(\prod_{j=1}^{n} \prod_{k=1}^{r}\left(a_{i j}^{(k *)}\right)^{\lambda_{k}}\right)^{1 / n}=\prod_{k=1}^{r}\left(\prod_{j=1}^{n} a_{i j}^{(k *)}\right)^{\lambda_{k} / n}, \quad i \in\{1,2, \ldots, n\}
$$

Under AIP, the individual priority vectors derived from the nearest consistency matrices $A^{(k *)}=\left(a_{i j}^{(k *)}\right)_{n \times n}(k=1,2, \ldots, r)$ by any prioritization method (Here, use LLSM) are $V^{(k *)}=\left(v_{1}^{(k *)}, v_{2}^{(k *)}, \ldots, v_{n}^{(k *)}\right)^{T}$, where $v_{i}^{(k *)}=\left(\prod_{j=1}^{n} a_{i j}^{(k *)}\right)^{1 / n}, i \in\{1,2, \ldots, n\}$. The group priority vector obtained by WGMM is $V^{G 2}=\left(v_{1}^{G 2}, v_{2}^{G 2}, \ldots, v_{n}^{G 2}\right)^{T}$, where

$$
v_{i}^{G 2}=\prod_{k=1}^{r}\left(\left(\prod_{j=1}^{n} a_{i j}^{(k *)}\right)^{1 / n}\right)^{\lambda_{k}}=\prod_{k=1}^{r}\left(\prod_{j=1}^{n} a_{i j}^{(k *)}\right)^{\lambda_{k} / n}, \quad i \in\{1,2, \ldots, n\}
$$

From the above expressions (13) and (14), it follows that $v_{i}^{G 1}=v_{i}^{G 2}$ for all $i \in$ $\{1,2, \ldots, n\}$.

Theorem 7 Let $V^{(k)}=\left(v_{1}^{(k)}, v_{2}^{(k)}, \ldots, v_{n}^{(k)}\right)^{T}$ be the individual priority vectors derived from the individual PCMs $A^{(k)}=\left(a_{i j}^{(k)}\right)_{n \times n}(k=1,2, \ldots, r)$ by certain prioritization method, and $V^{G}=\left(v_{1}^{G}, v_{2}^{G}, \ldots, v_{n}^{G}\right)^{T}$ be the group priority vector obtained by ANCM. If $v_{i}^{(k)}>v_{l}^{(k)}$ for all $k \in\{1,2, \ldots, r\}$, then $v_{i}^{G}>v_{l}^{G}$.

Proof of Theorem 7 is straightforward, we omit it here.

From Theorem 6, the group priority vectors obtained by AIJ and AIP based on the nearest consistency matrices are always identical, regardless of prioritization method, thus, the disputes on selecting AIJ or AIP are avoided when selecting ANCM as aggregation method in AHP-GDM. From Theorem 7, the results of AHP-GDM obtained by ANCM do not violate the Pareto principle of social choice theory (The Pareto principle essentially says that given two alternatives $\mathrm{A}$ and $\mathrm{B}$, if each member of a group of individuals prefers $\mathrm{A}$ to $\mathrm{B}$, then the group must prefer A to B).

It is a valid way to replace the individual PCM by a positive reciprocal matrix with the acceptable consistency and consensus when which differs from the individual PCM as little as possible according to certain metric such as Euclidean distance and similarity measure. Thus, the group priority vector is easily obtained by ANCM in AHP-GDM, the involved steps are briefly described as follows:

Step 1 Construct the individual PCMs $A^{(k)}=\left(a_{i j}^{(k)}\right)_{n \times n}(k=1,2, \ldots, r)$ by decision makers under one to nine scale, where $a_{i j}^{(k)}>0, a_{i i}^{(k)}=1$ and $a_{i j}^{(k)}=1 / a_{j i}^{(k)}$;

Step 2 Check the consensus of the individual PCMs according to GCCI and GOCI, and then assume that $A^{\left(k_{1}\right)}=\left(a_{i j}^{\left(k_{1}\right)}\right)_{n \times n}\left(k_{1}=1,2, \ldots, l\right)$ denote the individual PCMs without the acceptable consensus while $A^{\left(k_{2}\right)}=\left(a_{i j}^{\left(k_{2}\right)}\right)_{n \times n}\left(k_{2}=1,2, \ldots, r-l\right)$ denote the individual PCMs with the acceptable consensus; 
Step 3 Calculate the individual revision matrices $\hat{A}^{\left(k_{1}\right)}=\left(\hat{a}_{i j}^{\left(k_{1}\right)}\right)_{n \times n}\left(k_{1}=1,2, \ldots, l\right)$ of $A^{\left(k_{1}\right)}=\left(a_{i j}^{\left(k_{1}\right)}\right)_{n \times n}$, where $\hat{a}_{i j}^{\left(k_{1}\right)}>0, \hat{a}_{i i}^{\left(k_{1}\right)}=1$ and $\hat{a}_{i j}^{\left(k_{1}\right)}=1 / \hat{a}_{j i}^{\left(k_{1}\right)}\left(k_{1}=1,2, \ldots, l\right)$, and then assume that $\hat{A}^{(k)}=\left(\hat{a}_{i j}^{(k)}\right)_{n \times n}(k=1,2, \ldots, r)$ denote $\hat{A}^{\left(k_{1}\right)}=\left(\hat{a}_{i j}^{\left(k_{1}\right)}\right)_{n \times n}\left(k_{1}=1,2, \ldots, l\right)$ and $A^{\left(k_{2}\right)}=\left(a_{i j}^{\left(k_{2}\right)}\right)_{n \times n}\left(k_{2}=1,2, \ldots, r-l\right)$;

Step 4 Calculate the transitional matrices $X^{(k)}=\left(x_{i j}^{(k)}\right)_{n \times n}$ of $\hat{A}^{(k)}=\left(\hat{a}_{i j}^{(k)}\right)_{n \times n}(k=$ $1,2, \ldots, r)$, where $x_{i j}^{(k)}=\frac{1}{n} \ln \left(\prod_{l=1}^{n} \hat{a}_{i l}^{(k)} / \hat{a}_{j l}^{(k)}\right)(i<j), x_{i j}^{(k)}=-x_{j i}^{(k)}(i>j), x_{i i}=0$;

Step 5 Calculate the nearest consistency matrices $A^{(k *)}=\left(a_{i j}^{(k *)}\right)_{n \times n}(k=1,2, \ldots, r)$ of $\hat{A}^{(k)}=\left(\hat{a}_{i j}^{(k)}\right)_{n \times n}$, where $a_{i j}^{(k *)}=\exp \left(x_{i j}^{(k)}\right)(i<j), a_{i j}^{(k *)}=1 / a_{j i}^{(k *)}(i>j), a_{i i}^{(k *)}=1$;

Step 6 Calculate the consistent group matrix $A^{G}=\left(a_{i j}^{G}\right)_{n \times n}$ by WGMM based on $A^{(k *)}=$ $\left(a_{i j}^{(k *)}\right)_{n \times n}(k=1,2, \ldots, r)$, where $a_{i j}^{G}=\prod_{k=1}^{r}\left(a_{i j}^{(k *)}\right)^{\lambda_{k}}(i<j), a_{i j}^{G}=1 / a_{j i}^{G}(i>j)$, $a_{i i}^{G}=1$;

Step 7 Derive the unique group priority vector $V^{G}=\left(v_{1}^{G}, v_{2}^{G}, \ldots, v_{n}^{G}\right)^{T}$ by prioritization method (LLSM), where $v_{i}^{G}=\left(\prod_{j=1}^{n} a_{i j}^{G}\right)^{1 / n}=\prod_{k=1}^{r}\left(\prod_{j=1}^{n} a_{i j}^{(k *)}\right)^{\lambda_{k} / n}(i=1,2, \ldots, n)$.

In AHP-GDM, we first revise the individual PCMs without the acceptable consensus and then obtain the individual revision matrices; We then calculate the corresponding nearest consistency matrices of the individual revision matrices and the original individual PCMs with the acceptable consensus; We finally aggregate the nearest consistency matrices to obtain a consistent group matrix and then derive the unique group priority vector by any of prioritization methods such as ANM, EM, LLSM, etc.

\section{Numerical examples}

In this section, we illustrate the applications and advantages of the proposed ANCM by two numerical examples. Note that we take LLSM as prioritization method in ANCM, and adopt OC as consensus tool to revise the individual PCMs without the acceptable consensus. We assume that the weight of decision makers is equal since the nearest consistency matrices are of the acceptable consensus.

Example 1 Let us consider the group decision analysis situation with six decision makers taken from Wang and Xu (1990) and Altuzarra et al. (2007). With regards to the same decision problem, six individual PCMs $A^{(k)}(k=1,2, \ldots, 6)$ provided by six decision makers are given as follows: 
Table 1 GCCIs and GOCIs of six individual PCMs and two individual revision matrices

\begin{tabular}{lllllllll}
\hline Matrix & $A^{(1)}$ & $A^{(2)}$ & $A^{(3)}$ & $A^{(4)}$ & $A^{(5)}$ & $A^{(6)}$ & $A^{\prime(3)}$ & $A^{\prime(4)}$ \\
\hline GCCI & 0.113 & 0.378 & 0.693 & 0.752 & 0.338 & 0.045 & 0.391 & 0.353 \\
GOCI & 0 & 0 & 0.4 & 0.8 & 0 & 0 & 0 & 0.4
\end{tabular}

Table 2 Priority vectors and ranking orders of six individual PCMs and two individual revision matrices by EM

\begin{tabular}{lllllllll}
\hline Matrix & $A^{(1)}$ & $A^{(2)}$ & $A^{(3)}$ & $A^{(4)}$ & $A^{(5)}$ & $A^{(6)}$ & $A^{\prime(3)}$ & $A^{\prime(4)}$ \\
\hline$v_{1}^{(k)}$ & $0.493(1)$ & $0.489(1)$ & $0.278(2)$ & $0.466(1)$ & $0.388(1)$ & $0.474(1)$ & $0.373(1)$ & $0.443(1)$ \\
$v_{2}^{(k)}$ & $0.231(2)$ & $0.250(2)$ & $0.308(1)$ & $0.164(3)$ & $0.308(2)$ & $0.261(2)$ & $0.247(2)$ & $0.195(2)$ \\
$v_{3}^{(k)}$ & $0.093(4)$ & $0.113(4)$ & $0.119(4)$ & $0.209(2)$ & $0.080(4)$ & $0.098(4)$ & $0.146(4)$ & $0.153(4)$ \\
$v_{4}^{(k)}$ & $0.137(3)$ & $0.116(3)$ & $0.198(3)$ & $0.081(4)$ & $0.154(3)$ & $0.121(3)$ & $0.173(3)$ & $0.155(3)$ \\
$v_{5}^{(k)}$ & $0.047(5)$ & $0.032(5)$ & $0.097(5)$ & $0.078(5)$ & $0.070(5)$ & $0.046(5)$ & $0.061(5)$ & $0.053(5)$ \\
\hline
\end{tabular}

$$
\begin{aligned}
& A^{(1)}=\left[\begin{array}{ccccc}
1 & 3 & 5 & 4 & 7 \\
1 / 3 & 1 & 3 & 2 & 5 \\
1 / 5 & 1 / 3 & 1 & 1 / 2 & 3 \\
1 / 4 & 1 / 2 & 2 & 1 & 3 \\
1 / 7 & 1 / 5 & 1 / 3 & 1 / 3 & 1
\end{array}\right], A^{(2)}=\left[\begin{array}{ccccc}
1 & 4 & 3 & 5 & 8 \\
1 / 4 & 1 & 4 & 3 & 6 \\
1 / 3 & 1 / 4 & 1 & 1 & 5 \\
1 / 5 & 1 / 3 & 1 & 1 & 7 \\
1 / 8 & 1 / 6 & 1 / 5 & 1 / 7 & 1
\end{array}\right], \\
& A^{(3)}=\left[\begin{array}{ccccc}
1 & 1 / 2 & 3 & 2 & 5 \\
2 & 1 & 5 & 1 & 2 \\
1 / 3 & 1 / 5 & 1 & 2 & 1 / 2 \\
1 / 2 & 1 & 1 / 2 & 1 & 5 \\
1 / 5 & 1 / 2 & 2 & 1 / 5 & 1
\end{array}\right] \\
& A^{(4)}=\left[\begin{array}{ccccc}
1 & 3 & 5 & 2 & 6 \\
1 / 3 & 1 & 1 & 3 & 2 \\
1 / 5 & 1 & 1 & 4 & 5 \\
1 / 2 & 1 / 3 & 1 / 4 & 1 & 1 / 2 \\
1 / 6 & 1 / 2 & 1 / 5 & 2 & 1
\end{array}\right] \\
& A^{(5)}=\left[\begin{array}{ccccc}
1 & 2 & 6 & 3 & 3 \\
1 / 2 & 1 & 2 & 5 & 4 \\
1 / 6 & 1 / 2 & 1 & 1 / 2 & 1 \\
1 / 3 & 1 / 5 & 2 & 1 & 5 \\
1 / 3 & 1 / 4 & 1 & 1 / 5 & 1
\end{array}\right], A^{(6)}=\left[\begin{array}{ccccc}
1 & 2 & 5 & 4 & 9 \\
1 / 2 & 1 & 3 & 2 & 6 \\
1 / 5 & 1 / 3 & 1 & 1 & 2 \\
1 / 4 & 1 / 2 & 1 & 1 & 3 \\
1 / 9 & 1 / 6 & 1 / 2 & 1 / 3 & 1
\end{array}\right] .
\end{aligned}
$$

We first consider the consensus of six individual PCMs. GCCIs and GOCIs of six individual PCMs are shown in Table 1. The priority vectors and ranking orders derived from six individual PCMs by EM are shown in Table 2 (Thereinafter, the numbers in parentheses are ranking orders).

From Table 1, we have that

$$
\operatorname{GCCI}\left(A^{(3)}\right)>0.5, \operatorname{GOCI}\left(A^{(3)}\right)>0 ; G \operatorname{CCI}\left(A^{(4)}\right)>0.5, \operatorname{GOCI}\left(A^{(4)}\right)>0 .
$$


Table 3 Priority vectors and ranking orders of six nearest consistency matrices

\begin{tabular}{lllllll}
\hline Matrix & $A^{(1 *)}$ & $A^{(2 *)}$ & $A^{(3 *)}$ & $A^{(4 *)}$ & $A^{(5 *)}$ & $A^{(6 *)}$ \\
\hline$v_{1}^{(k)}$ & $0.491(1)$ & $0.481(1)$ & $0.405(1)$ & $0.453(1)$ & $0.407(1)$ & $0.475(1)$ \\
$v_{2}^{(k)}$ & $0.232(2)$ & $0.250(2)$ & $0.219(2)$ & $0.184(2)$ & $0.291(2)$ & $0.261(2)$ \\
$v_{3}^{(k)}$ & $0.092(4)$ & $0.117(4)$ & $0.137(4)$ & $0.156(3)$ & $0.085(4)$ & $0.098(4)$ \\
$v_{4}^{(k)}$ & $0.138(3)$ & $0.120(3)$ & $0.176(3)$ & $0.155(4)$ & $0.147(3)$ & $0.120(3)$ \\
$v_{5}^{(k)}$ & $0.046(5)$ & $0.032(5)$ & $0.063(5)$ & $0.053(5)$ & $0.070(5)$ & $0.046(5)$ \\
\hline
\end{tabular}

That is, $A^{(3)}$ and $A^{(4)}$ are not of the acceptable consensus and should be revised to reach the acceptable consensus. From Table 2, the preference structures of $A^{(3)}$ and $A^{(4)}$ are (21435) and (13245), respectively, which are in disagreement with the collective preference structure (12435) supported by the other four individual PCMs. Thus $A^{(3)}$ and $A^{(4)}$ are not of the fully ordinal consensus and should be adjusted. Here, we directly adopt OC as consensus tool in the process of adjusting $A^{(3)}$ and $A^{(4)}$, since OC can find the judgments disagreeing with the collective preference structure preferred by the other decision makers. The judgments found by OC should be dealt with to reach the acceptable consensus. According to $\mathrm{OC}$, the judgments with the highest levels of variability in $A^{(3)}$ and $A^{(4)}$ are $a_{12}, a_{34}, a_{35}$ and $a_{45}$ since $O C_{12}=8, O C_{34}=8, O C_{35}=10$ and $O C_{45}=14$. Moreover, the judgments $\left(a_{12}^{(3)}, a_{35}^{(3)}, a_{34}^{(4)}\right.$ and $\left.a_{45}^{(4)}\right)$ disagree with the collective preference structure (12435) supported by the other four individual PCMs. For reaching the acceptable consensus, we replace $a_{i j}^{(k)}$ by $a_{i j}^{(k)}=\left(\prod_{l \neq k} a_{i j}^{(l)}\right)^{\frac{1}{r-1}}(k=3,4)$ for $a_{12}^{(3)}, a_{35}^{(3)}, a_{34}^{(4)}$ and $a_{45}^{(4)}$ in $A^{(3)}$ and $A^{(4)}$. Thereby, $a_{12}^{\prime(3)}=2.702, a_{35}^{\prime(3)}=2.724, a_{34}^{\prime(4)}=0.871$ and $a_{45}^{\prime(4)}=4.360$. Accordingly, $a_{21}^{\prime(3)}=0.370$, $a_{53}^{\prime(3)}=0.367, a_{43}^{\prime(4)}=1.149$ and $a_{54}^{\prime(4)}=0.229$. Two individual revision matrices $\left(A^{\prime(3)}\right.$ and $\left.A^{\prime(4)}\right)$ are listed as below. GCCIs and GOCIs of $A^{\prime(3)}$ and $A^{\prime(4)}$ are also shown in Table 1.

$$
A^{\prime(3)}=\left[\begin{array}{ccccc}
1 & 2.702 & 3 & 2 & 5 \\
0.370 & 1 & 5 & 1 & 2 \\
1 / 3 & 1 / 5 & 1 & 2 & 2.724 \\
1 / 2 & 1 & 1 / 2 & 1 & 5 \\
1 / 5 & 1 / 2 & 0.367 & 1 / 5 & 1
\end{array}\right], A^{\prime(4)}=\left[\begin{array}{ccccc}
1 & 3 & 5 & 2 & 6 \\
1 / 3 & 1 & 1 & 3 & 2 \\
1 / 5 & 1 & 1 & 0.871 & 5 \\
1 / 2 & 1 / 3 & 1.149 & 1 & 4.360 \\
1 / 6 & 1 / 2 & 1 / 5 & 0.229 & 1
\end{array}\right] .
$$

From Table 1, we have that

$$
\begin{aligned}
& \operatorname{GCCI}\left(A^{\prime(3)}\right)<0.5<\operatorname{GCCI}\left(A^{(3)}\right), \operatorname{GOCI}\left(A^{\prime(3)}\right)=0<\operatorname{GOCI}\left(A^{(3)}\right) ; \\
& \operatorname{GCC} I\left(A^{\prime(4)}\right)<0.5<\operatorname{GCCI}\left(A^{(4)}\right), \operatorname{GOCI}\left(A^{\prime(4)}\right)=0.4(0)<\operatorname{GOCI}\left(A^{(4)}\right) .
\end{aligned}
$$

That is, the individual revision matrix $A^{\prime(3)}$ reaches the acceptable consensus. For individual revision matrix $A^{\prime(4)}$, the distance between $0.156(3)$ and $0.155(4)$ is 0.001 (very little), thus the rank orders of them can be exchanged by coordination, thereby GOCI of $A^{\prime(4)}$ is equal to zero. That is, $A^{\prime(4)}$ can reach the fully ordinal consensus by coordination. Thus, $A^{\prime(3)}$ and $A^{\prime(4)}$ are of the acceptable consensus.

We next achieve the nearest consistency matrices of four original individual PCMs $\left(A^{(1)}\right.$, $\left.A^{(2)}, A^{(5)}, A^{(6)}\right)$ and two individual revision matrices $\left(A^{\prime(3)}, A^{\prime(4)}\right)$ using Two-step algorithm, which are denoted by $A^{(k *)}(k=1,2, \ldots, 6)$. The priority vector derived from a nearest consistency matrix is unique, regardless of prioritization method. Thus, the priority vectors from the nearest consistency matrices derived by any of prioritization methods are shown in Table 3. 
Table 4 Group priority vectors and ranking orders obtained by ANCM, BPP, AIP and AIJ
Table 5 GCIs and PVNs of ANCM, BPP, AIP and AIJ

\begin{tabular}{lllll}
\hline Aggregation method & ANCM & BPP & AIP(EM) & AIJ(EM) \\
\hline$\hat{v}_{1}^{(k)}$ & $0.455(1)$ & $0.479(1)$ & $0.436(1)$ & $0.438(1)$ \\
$\hat{v}_{2}^{(k)}$ & $0.239(2)$ & $0.259(2)$ & $0.256(2)$ & $0.258(2)$ \\
$\hat{v}_{3}^{(k)}$ & $0.113(4)$ & $0.097(4)$ & $0.116(4)$ & $0.114(4)$ \\
$\hat{v}_{4}^{(k)}$ & $0.143(3)$ & $0.119(3)$ & $0.133(3)$ & $0.130(3)$ \\
$\hat{v}_{5}^{(k)}$ & $0.051(5)$ & $0.045(5)$ & $0.059(5)$ & $0.059(5)$ \\
\hline
\end{tabular}

\begin{tabular}{lllll}
\hline Aggregation method & ANCM & BPP & AIP(EM) & AIJ(EM) \\
\hline GCI & 0.249 & 0.363 & 0.387 & 0.387 \\
PVN & 0.058 & 0.108 & 0.108 & 0.108 \\
\hline
\end{tabular}

We finally compare ANCM with BPP (Altuzarra et al. 2007), AIP and AIJ according to two compatibility indicators (GCI and PVN). The group priority vectors and ranking orders derived by ANCM, BPP, AIP and AIJ are shown in Table 4 (We consider EM as prioritization method in AIP and AIJ). The compatibility indicators (GCI and PVN) between the individual judgments and the group priority vectors obtained by ANCM, BPP, AIP and AIJ are shown in Table 5.

From Tables 2 and 4, the ranking orders derived from four original individual PCMs $\left(A^{(1)}, A^{(2)}, A^{(5)}, A^{(6)}\right)$ and two individual revision matrices $\left(A^{\prime(3)}, A^{\prime(4)}\right)$ are exactly the same as the group ranking order obtained by ANCM. It is concluded that ANCM does not violate the Pareto principal of social choice theory in Example 1. From Table 4, the collective preference structure (12435) obtained by ANCM is the same as the ones obtained by BPP, AIP and AIJ although the group priority vectors obtained by them are different. From Table 5, GCI and PVN of ANCM are less than GCIs and PVNs of BPP, AIP and AIJ, respectively. With respect to the ordinal compatibility and cardinal compatibility, the result of ANCM is more reliable than those of BPP, AIP and AIJ in AHP-GDM.

Example 2 Let us consider the group decision analysis example included in Dong et al. (2010), which consists of five decision makers and four alternatives. Five individual PCMs $A^{(k)}(k=1,2, \ldots, 5)$ are listed below:

$$
\begin{aligned}
A^{(1)} & =\left[\begin{array}{cccc}
1 & 4 & 6 & 7 \\
1 / 4 & 1 & 3 & 4 \\
1 / 6 & 1 / 3 & 1 & 2 \\
1 / 7 & 1 / 4 & 1 / 2 & 1
\end{array}\right], A^{(2)}=\left[\begin{array}{cccc}
1 & 5 & 7 & 9 \\
1 / 5 & 1 & 4 & 6 \\
1 / 7 & 1 / 4 & 1 & 2 \\
1 / 9 & 1 / 6 & 1 / 2 & 1
\end{array}\right], A^{(3)}=\left[\begin{array}{cccc}
1 & 3 & 5 & 8 \\
1 / 3 & 1 & 4 & 5 \\
1 / 5 & 1 / 4 & 1 & 2 \\
1 / 8 & 1 / 5 & 1 / 2 & 1
\end{array}\right], \\
A^{(4)} & =\left[\begin{array}{cccc}
1 & 4 & 5 & 6 \\
1 / 4 & 1 & 3 & 3 \\
1 / 5 & 1 / 3 & 1 & 2 \\
1 / 6 & 1 / 3 & 1 / 2 & 1
\end{array}\right], A^{(5)}=\left[\begin{array}{cccc}
1 & 1 / 2 & 1 & 2 \\
2 & 1 & 1 / 2 & 3 \\
1 & 2 & 1 & 4 \\
1 / 2 & 1 / 3 & 1 / 4 & 1
\end{array}\right] ; A^{(5)} \\
& =\left[\begin{array}{cccc}
1 & 3.963 & 5.692 & 7.416 \\
0.254 & 1 & 3.464 & 3 \\
0.176 & 0.289 & 1 & 4 \\
0.135 & 1 / 3 & 1 / 4 & 1
\end{array}\right] .
\end{aligned}
$$


Table 6 GCCIs and GOCIs of five individual PCMs and an individual revision matrix

Table 7 Priority vectors and ranking orders of five individual PCMs and an individual revision matrix by EM

\begin{tabular}{lllllll}
\hline Matrix & $A^{(1)}$ & $A^{(2)}$ & $A^{(3)}$ & $A^{(4)}$ & $A^{(5)}$ & $A^{\prime(5)}$ \\
\hline GCCI & 0.139 & 0.320 & 0.135 & 0.134 & 1.334 & 0.253 \\
GOCI & 0 & 0 & 0 & 0 & 1 & 0 \\
\hline
\end{tabular}

\begin{tabular}{lllllll}
\hline Matrix & $A^{(1)}$ & $A^{(2)}$ & $A^{(3)}$ & $A^{(4)}$ & $A^{(5)}$ & $A^{\prime(5)}$ \\
\hline$v_{1}^{(k)}$ & $\begin{array}{c}0.617 \\
(1)\end{array}$ & $0.653(1)$ & $0.570(1)$ & $0.597(1)$ & $0.228(3)$ & $0.601(1)$ \\
$v_{2}^{(k)}$ & $0.224(2)$ & $0.225(2)$ & $0.277(2)$ & $0.222(2)$ & $0.295(2)$ & $0.223(2)$ \\
$v_{3}^{(k)}$ & $0.097(3)$ & $0.076(3)$ & $0.096(3)$ & $0.108(3)$ & $0.380(1)$ & $0.120(3)$ \\
$v_{4}^{(k)}$ & $0.062(4)$ & $0.047(4)$ & $0.057(4)$ & $0.073(4)$ & $0.097(4)$ & $0.056(4)$
\end{tabular}

We first consider the consensus of five individual PCMs. GCCIs and GOCIs of five individual PCMs are shown in Table 6 . The priority vectors and ranking orders derived from five individual PCMs by EM are shown in Table 7.

From Table 6, we have

$$
\operatorname{GCCI}\left(A^{(5)}\right)>0.5, \operatorname{GOCI}\left(A^{(5)}\right)>0
$$

That is, only $A^{(5)}$ is not of the acceptable consensus. From Table 7, the preference structure (3214) of $A^{(5)}$ is different with the collective preference structure (1234) supported by the other four individual PCMs, that is, $A^{(5)}$ is not of the fully ordinal consensus. Thus, $A^{(5)}$ should be adjusted to reach the acceptable consensus. Here, we still adopt OC as consensus tool to revise $A^{(5)}$. According to $\mathrm{OC}$, the judgments with the highest levels of variability in $A^{(5)}$ are $a_{12}, a_{13}, a_{14}$ and $a_{23}$ since $O C_{12}=10, O C_{13}=7, O C_{14}=4.5$ and $O C_{23}=8$. Moreover, the judgments $\left(a_{12}^{(5)}, a_{13}^{(5)}, a_{14}^{(5)}, a_{23}^{(5)}\right)$ disagree with the collective preference structure (1234) supported by the other four individual PCMs. For reaching the acceptable consensus, we replace $a_{i j}^{(5)}$ by $a_{i j}^{(5)}=\left(\prod_{l \neq k} a_{i j}^{(l)}\right)^{\frac{1}{r-1}}$ for $a_{12}^{(5)}, a_{13}^{(5)}, a_{14}^{(5)}$ and $a_{23}^{(5)}$ in $A^{(5)}$. Thereby, $a_{12}^{\prime(5)}=3.936, a_{13}^{\prime(5)}=5.692, a_{14}^{\prime(5)}=7.416$ and $a_{23}^{\prime(5)}=3.464$. Accordingly, $a_{21}^{\prime(5)}=0.254, a_{31}^{\prime(5)}=0.176, a_{41}^{\prime(5)}=0.135$ and $a_{32}^{\prime(5)}=0.289$. The individual revision matrix $A^{\prime(5)}$ is listed up. Moreover, GCCI and GOCI of $A^{\prime(5)}$ are shown in Table 6. The priority vector and ranking order derived from $A^{\prime(5)}$ by EM are shown in Table 7. From Table 6, we have that

$$
\operatorname{GCCI}\left(A^{\prime(5)}\right)<0.5<\operatorname{GCCI}\left(A^{(5)}\right), \operatorname{GOCI}\left(A^{\prime(5)}\right)=0<\operatorname{GOCI}\left(A^{(5)}\right) .
$$

It is concluded that $A^{\prime(5)}$ is of the acceptable cardinal consensus and is of fully ordinal consensus. That is, $A^{\prime(5)}$ is of the acceptable consensus.

We next achieve the nearest consistency matrices for four original individual PCMs $\left(A^{(1)}\right.$, $\left.A^{(2)}, A^{(3)}, A^{(4)}\right)$ and an individual revision matrix $\left(A^{\prime(5)}\right)$ using two-step algorithm, which are denoted by $A^{(k *)}(k=1,2, \ldots, 5)$. The priority vectors from the nearest consistency matrices derived by any of prioritization methods are shown in Table 8.

We finally compare ANCM with RGMM (Dong et al. 2010), AIP and AIJ according to two compatibility indicators (GCI and PVN). The group priority vectors and ranking orders obtained by ANCM, RGMM, AIP and AIJ are shown in Table 9 (We consider EM as 
Table 8 Priority vectors and ranking orders of five nearest consistency matrices

\begin{tabular}{llllll}
\hline Matrix & $A^{(1 *)}$ & $A^{(2 *)}$ & $A^{(3 *)}$ & $A^{(4 *)}$ & $A^{(5 *)}$ \\
\hline$v_{1}^{(k)}$ & $0.614(1)$ & $0.646(1)$ & $0.569(1)$ & $0.597(1)$ & $0.613(1)$ \\
$v_{2}^{(k)}$ & $0.225(2)$ & $0.227(2)$ & $0.276(2)$ & $0.221(2)$ & $0.217(2)$ \\
$v_{3}^{(k)}$ & $0.099(3)$ & $0.079(3)$ & $0.097(3)$ & $0.109(3)$ & $0.114(3)$ \\
$v_{4}^{(k)}$ & $0.062(4)$ & $0.048(4)$ & $0.058(4)$ & $0.074(4)$ & $0.056(4)$ \\
\hline
\end{tabular}

\begin{tabular}{lllll}
\hline Aggregation method & ANCM & RGMM & AIP(EM) & AIJ(EM) \\
\hline$\hat{v}_{1}^{(k)}$ & $0.6092(1)$ & $0.5416(1)$ & $0.5344(1)$ & $0.5324(1)$ \\
$\hat{v}_{2}^{(k)}$ & $0.2329(2)$ & $0.2579(2)$ & $0.2637(2)$ & $0.2634(2)$ \\
$\hat{v}_{3}^{(k)}$ & $0.0991(3)$ & $0.1299(3)$ & $0.1326(3)$ & $0.1333(3)$ \\
$\hat{v}_{4}^{(k)}$ & $0.0589(4)$ & $0.0688(4)$ & $0.0694(4)$ & $0.0709(4)$ \\
\hline
\end{tabular}

Table 9 Group priority vectors and ranking orders obtained by ANCM, BPP, AIP and AIJ

Table 10 GCIs and PVNs of ANCM, BPP, AIP and AIJ

\begin{tabular}{lllll}
\hline Aggregation method & ANCM & RGMM & AIP(EM) & AIJ(EM) \\
\hline GCI & 0.127 & 0.341 & 0.415 & 0.391 \\
PVN & 0.000 & 0.083 & 0.083 & 0.083 \\
\hline
\end{tabular}

prioritization method in AIP and AIJ). The compatibility indicators (GCI and PVN) between the individual judgments and the group priority vectors obtained by ANCM, RGMM, AIP and AIJ are shown in Table 10.

From Tables 7 and 9, the ranking orders derived from four original individual PCMs $\left(A^{(1)}, A^{(2)}, A^{(3)}, A^{(4)}\right)$ and an individual revision matrix $\left(A^{\prime(5)}\right)$ are exactly the same as the group ranking order obtained by ANCM. It is concluded that ANCM does not violate the Pareto principal of social choice theory in Example 2. From Table 9, the collective preference structure (1234) obtained by ANCM is the same as the ones obtained by RGMM, AIP and AIJ although the group priority vectors obtained by them are different. From Table 10, GCI and PVN of ANCM are less than GCIs and PVNs of RGMM, AIP and AIJ, respectively. With respect to the ordinal compatibility and cardinal compatibility, the result of ANCM is more reliable than those of RGMM, AIP and AIJ in AHP-GDM.

The above two examples illustrate how the proposed ANCM performs and the advantages of ANCM in AHP-GDM. The results show that ANCM more reflects the opinions of the group as shown by the acceptable consistency and consensus as well as the higher compatibility indicators for the majority of decision makers and results in the better cardinal compatibility and ordinal compatibility than other aggregation methods such as BPP, RGMM, AIJ and AIP.

\section{Conclusions}

In this paper, we propose aggregation of the nearest consistency matrices (ANCM) with the acceptable consensus, simultaneously considering the consensus and consistency of the individual PCMs in AHP-GDM. Two numerical examples are examined to illustrate the applications and advantages of the proposed ANCM. The main characteristics of ANCM are 
as follows: (1) It is independent of prioritization methods used in AHP-GDM; (2) It results in the same group ranking order as BPP, RGMM, AIJ and AIP; (3) It does not violate Pareto principle of social choice theory; (4) It effectively improves the consensus and consistency of the individual PCMs and the compatibility. Moreover, the proposed ANCM is easy to program and implement in resolving highly complex group decision making problems while resolving some real-world situations in economy and management fields. In the future research, we will further extend ANCM to the incomplete and imprecise individual PCMs in AHP-GDM.

Acknowledgements This research has been partially supported by Grants from Humanities and Social Science Program of Ministry of Education of China (\#17YJA630051) and Grants from the National Natural Science Foundation of China (\#U1811462, \#71725001, and \#71471149).

Open Access This article is licensed under a Creative Commons Attribution 4.0 International License, which permits use, sharing, adaptation, distribution and reproduction in any medium or format, as long as you give appropriate credit to the original author(s) and the source, provide a link to the Creative Commons licence, and indicate if changes were made. The images or other third party material in this article are included in the article's Creative Commons licence, unless indicated otherwise in a credit line to the material. If material is not included in the article's Creative Commons licence and your intended use is not permitted by statutory regulation or exceeds the permitted use, you will need to obtain permission directly from the copyright holder. To view a copy of this licence, visit http://creativecommons.org/licenses/by/4.0/.

\section{References}

Aczel, J., \& Saaty, T. L. (1983). Procedures for synthesizing ratio judgments. Journal of Mathematical Psychology, 27(1), 93-102.

Altuzarra, A., Moreno-Jiménez, J. M., \& Salvador, M. (2007). A Bayesian priorization procedure for AHPgroup decision making. European Journal of Operational Research, 182(1), 367-382.

Altuzarra, A., Moreno-Jiménez, J. M., \& Salvador, M. (2010). Consensus building in AHP-group decision making: A Bayesian approach. Operations Research, 58(6), 1755-1773.

Arrow, K. J. (1963). Social choice and individual values (2nd ed.). New York: Wiley.

Barzilai, J., \& Golany, B. (1994). AHP rank reversal normalization and aggregation rules. INFOR, 32(2), 57-64.

Benítez, J., Delgado-Galván, X., Izquierdo, J., \& Pérez-García, R. (2011). Achieving matrix consistency in AHP through linearization. Applied Mathematical Modelling, 35(9), 4449-4457.

Benítez, J., Izquierdo, J., Pérez-García, R., \& Ramos-Martínez, E. (2014). A simple formula to find the closest consistent matrix to a reciprocal matrix. Applied Mathematical Modelling, 38(15-16), 3968-3974.

Crawford, G., \& Williams, C. (1985). A note on the analysis of subjective judgment matrices. Journal of Mathematical Psychology, 29(4), 387-405.

Dong, Q., \& Saaty, T. L. (2014). An analytic hierarchy process model of group gonsensus. Journal of Systems Science and Systems Engineering, 23(3), 362-374.

Dong, Y. C., Zhang, G. Q., Hong, W. C., \& Xu, Y. F. (2010). Consensus models for AHP group decision making under row geometric mean prioritization method. Decision Support Systems, 49(3), 281-289.

Escobar, M. T., \& Moreno-Jimenez, J. M. (2007). Aggregation of individual preference structures in AHPgroup decision making. Group Decision and Negotiation, 16(4), 287-301.

Forman, E., \& Peniwati, K. (1998). Aggregating individual judgments and priorities with the analytic hierarchy process. European Journal of Operational Research, 108(1), 165-169.

Gargallo, P., Moreno-Jiménez, J. M., \& Salvador, M. (2007). AHP-group decision making: A Bayesian approach based on mixtures for group pattern identification. Group Decision and Negotiation, 16(6), 485-506.

Golany, B., \& Kress, M. A. (1993). Multicriteria evaluation of methods for obtaining weights from ratio-scale matrices. European Journal of Operational Research, 69(2), 210-220.

Koczkodaj, W. W., \& Orlowski, M. (1997). An orthogonal basis for computing a consistent approximation to a pairwise comparisons matrix. Computers and Mathematics with Application, 34(10), 41-47.

Kou, G., Ergu, D., Chen, Y., \& Lin, C. (2016). Pairwise comparison matrix in multiple criteria decision making. Technological and Economic Development of Economy, 22(5), 738-765. 
Kou, G., \& Lin, C. (2014). A cosine maximization method for the priority vector derivation in AHP. European Journal of Operational Research, 235(1), 225-232.

Kou, G., Lu, Y., Peng, Y., \& Shi, Y. (2012). Evaluation of classification algorithms using MCDM and rank correlation. International Journal of Information Technology \& Decision Making, 11(1), 197-225.

Kou, G., Peng, Y., \& Wang, G. (2014). Evaluation of clustering algorithms for financial risk analysis using MCDM methods. Information Sciences, 275, 1-12.

Li, G., Kou, G., \& Peng, Y. (2018). A group decision making model for integrating heterogeneous information. IEEE Transactions on Systems, Man, and Cybernetics: Systems, 48, 982-992.

Li, Y., Zhang, H., \& Dong, Y. (2017). The interactive consensus reaching process with the minimum and uncertain cost in group decision making. Applied Soft Computing, 60, 202-212.

Lin, C., \& Kou, G. (2015). Bayesian revision of the individual pair-wise comparison matrices under consensus in AHP-GDM. Applied Soft Computing, 35(C), 802-811.

Lin, C., Kou, G., \& Ergu, D. (2013). A heuristic approach for deriving the priority vector in AHP. Applied Mathematical Modelling, 37(8), 5828-5836.

Meyer, C. D. (2000). Matrix analysis and applied linear algebra. Philadelphia: SIAM.

Moreno-Jiménez, J. M. (2011). An AHP/ANP multicriteria methodology to estimate the value and transfers fees of professional football players. In Proceedings ISAHP. Sorrento (Italia).

Ramanathan, R., \& Ganesh, L. S. (1994). Group preference aggregation methods employed in AHP: An evaluation and intrinsic process for deriving members' weightages. European Journal of Operational Research, 79(2), 249-265.

Saaty, T. L. (1977). A scaling method for priorities in hierarchical structures. Journal of Mathematical Psychology, 15(3), 234-281.

Saaty, T. L. (1980). The analytic hierarchy process. New York, NY: McGraw-Hill.

Saaty, T. L. (1989). Group decision-making and the AHP. In B. L. Golden, E. A. Wasil, \& P. T. Harker (Eds.), The analytic hierarchy process: Applications and studies (pp. 59-67). New York: Springer.

Srdjevic, B. (2005). Combining different prioritization methods in analytic hierarchy process synthesis. Computers \& Operations Research, 32, 1897-1919.

Srdjevic, B., \& Srdjevic, Z. (2013). Synthesis of individual best local priority vectors in AHP-group decision making. Applied Soft Computing, 13(4), 2045-2056.

Wang, Y. M., \& Xu, N. R. (1990). Linear programming method for solving the group-AHP. Journal of Southeast University (Natural Science Edition), 20, 58-63.

Wu, Z., \& Xu, J. (2012). A consistency and consensus based decision support model for group decision making with multiplicative preference relations. Decision Support Systems, 52(3), 757-767.

Zahir, S. (1999). Geometry of decision-making and the vector space formulation of the analytic hierarchy process. European Journal of Operational Research, 112(2), 373-396.

Zhang, H., Kou, G., \& Peng, Y. (2019). Soft consensus cost models for group decision making and economic interpretations. European Journal of Operational Research, 277(3), 964-980.

Publisher's Note Springer Nature remains neutral with regard to jurisdictional claims in published maps and institutional affiliations. 